\title{
Entre mobilités et immobilités
}

\section{Les frontières spatiales de la parenté dans les villages jàana (Burkina Faso)}

\author{
Delphine Manetta
}

Émulations - Revue de sciences sociales 2019, n 32, «Aux frontières de la parenté. Un éclairage par les marges ».

\section{Article disponible à l'adresse suivante}

https://ojs.uclouvain.be/index.php/emulations/article/view/manetta

\section{Pour citer cet article}

Delphine Manetta, « Entre mobilités et immobilités. Les frontières spatiales de la parenté dans les villages jàana (Burkina Faso) », Émulations, n³2, Mise en ligne le 20 avril 2020.

DOI : 10.14428/emulations.032.04

Distribution électronique : Université catholique de Louvain (Belgique) : ojs.uclouvain.be

(c) Cet article est mis à disposition selon les termes de la Licence Creative Commons Attribution, Pas d'Utilisation Commerciale 4.0 International. http://creativecommons.org/licenses/by-nc/4.0/

Éditeur : Émulations - Revue de sciences sociales / Presses universitaires de Louvain https://ojs.uclouvain.be/index.php/emulations

ISSN électronique : 1784-5734

PUL PRESSES UNIVERSITAIRES DE LOUVAIN 


\title{
Entre mobilités et immobilités
}

\author{
Les frontières spatiales de la parenté \\ dans les villages jàana (Burkina Faso)
}

Delphine Manetta ${ }^{1}$

\begin{abstract}
[Résumé] Fondé sur des recherches sur la mort dans des villages jàana du Sud-Ouest du Burkina Faso, cet article envisage les frontières de la parenté en « termes spatiaux ». II montre que ces frontières résultent de mobilités et d'immobilités féminines et masculines observables dans et depuis l'espace de la maison. Ces mobilités et ces immobilités font de la maison un lieu carrefour, qui se construit par des contacts et des mouvements questionnant la stabilité de l'alliance et, par-là même, la capacité de reproduction de la parenté dans l'espace et le temps. Elles ramifient l'intérieur de la maison à de multiples lieux extérieurs en situant la parenté dans un espace morcelé. La nature de ces lieux explique toutefois la localisation contingente des frontières spatiales de la parenté en dehors de l'enceinte domestique, mais aussi l'incertitude qui pèse toujours sur le devenir de la maison.
\end{abstract}

Mots-clés : frontière, espace, maison, mobilités, immobilités.

\section{Between mobilities and immobilities: \\ The spatial frontiers of kinship in jàana villages (Burkina Faso)}

[Abstract] Grounded on fieldwork research on death in Jàana villages in South West Burkina Faso, this article considers the frontiers of kinship in "spatial terms". It shows that these frontiers result from feminine and masculine mobilities and immobilities that can be observed inside and from the house. These mobilities and immobilities define the house as a crossroad: it is a place of contacts and movements where the stability of matrimonial alliance and the reproductive capacity of kinship in time and space are questioned. They connect the inside of the house to several external places, by situating kinship in a fragmented space. However, the nature of these places explains the contingent localization of the frontier of kinship outside the house, but also the uncertain future of the house.

Keywords: frontiers, space, house, mobilities, immobilities.

\section{Introduction}

Cet article ${ }^{2}$ aborde les frontières de la parenté par l'étude de l'étiologie de la mort dans des villages jàana (sing. jàan) ${ }^{3}$ du Sud-Ouest du Burkina Faso. Cette étiologie repose sur la recension, entre 2009 et 2015, de cinquante décès (de deux enfants, dix-sept femmes et trente et un hommes) dont les causes ont été dévoilées lors des rites funéraires,

\footnotetext{
${ }^{1}$ Université Paris Descartes, CANTHEL, France.

${ }^{2}$ Je remercie les évaluateurs et les relecteurs qui ont permis d'améliorer et de préciser le propos de cet article.

${ }^{3}$ Les Jàana forment un groupe voisin des Lobi, des Birifor, des Dagara et des Pougouli. Leur nom est écrit « Dyan » ou « Dian » dans la littérature coloniale et scientifique. J'ai choisi de transcrire leur appellation en langue jàan.
} 
notamment l'interrogatoire du cadavre mené par les chefs de terre des villages et la consultation divinatoire du rite de levée du deuil. L'interrogatoire du cadavre consiste à soumettre un mort, dont le corps ou son avatar (tels des vêtements) est enroulé dans une natte végétale et porté au sommet de la tête de deux fossoyeurs, à une série de questions : est-il coupable de sorcellerie ? A-t-il été tué par le dieu de la terre du village Giliyu ? L'ancêtre de son clan Posiiru ? Un dieu particulier auquel il a été initié ? Le cadavre répond en faisant se mouvoir les fossoyeurs, soit en faisant vaciller leur tête à gauche ou à droite pour signifier son refus ou son approbation, soit en se rendant devant l'objet ou la personne à l'origine du décès. La consultation divinatoire, qui se tient entre quinze et vingt jours après les premiers rites funéraires, permet de confirmer ou d'infirmer la cause du décès découverte pendant l'interrogatoire. Ratifiée par le sacrifice d'un poussin, cette consultation est essentielle parce que l'explication de la mort finalement retenue par le devin est reconnue comme la seule cause véritable du décès.

La lecture rétrospective de la mort réalisée pendant ces rites inscrit le décès dans l'histoire de celle ou celui qui meurt et, par extension, dans celle d'un groupe où l'intervention de dieux, d'ancêtres et de sorciers sanctionnent l'existence de conflits. En dépit de la pluralité apparente des causes de la mort, trois explications principales peuvent être repérées. Si les circulations monétaires (32\%) et la sorcellerie (11\%) sont les motifs d'un grand nombre de décès, l'alliance, prise dans son vécu et ses aléas, constitue la première cause de mort ( $47 \%)$ tous sexes confondus. Cet article s'intéresse spécifiquement aux morts liées à l'alliance afin de répondre à une question : dans quelle mesure érigent-elles la maison comme un carrefour au sein et à partir duquel se configurent les frontières spatiales de la parenté ? En étudiant la production de ces frontières à partir de la maison, cet article prolonge la perspective topologique initiée par Janet Carsten et Stephen Hugh-Jones (1995) et Klaus Hamberger (2010). En proposant une relecture de la notion de maison formulée par Claude Lévi-Strauss (1984, en particulier), ils ont redéfini la maison comme un épicentre social et spatial dont l'architecture objective l'identité de ceux qui y résident, leurs relations de parenté et leurs rapports aux autres groupes. Selon Hamberger, la maison se trouve ainsi au cœur d'une «théorie spatiale de l'alliance » (2010:11). La relation conjugale, en produisant des liens d'affinité et de filiation par la mise en relation de groupes échangistes, « constitue la maison de l'intérieur, en même temps qu'elle la lie à l'extérieur » (Hamberger, 2010 : 10). Ainsi proposet-il de concevoir l'alliance en « termes spatiaux » en transmuant les groupes donneur et receveur de femmes en " lieux », l'échange en « déplacement » et l'appartenance en identités résidentielles (Hamberger, 2010 : 10).

Cette lecture topologique interroge la dimension spatiale de la notion de frontière en modélisant les relations d'alliance et de parenté par des mouvements dans et depuis l'espace de la maison. La compréhension de la nature, de la direction et du sens de ces mouvements constitue le cœur de cet article. Il suggère que, pour identifier les frontières spatiales de la parenté, l'alliance doit être envisagée au travers des notions de mobilité et d'immobilité. Ces deux notions interdépendantes ont émergé des études 
sur les migrations (Reeves, 2011 ; Bergeon et al., 2013 ; Schewel, 2019) et ont rarement été appliquées à l'étude de la parenté à l'échelle de la résidence (Dessertine, 2016). Ce texte montrera que la mobilité et l'immobilité doivent être conçues comme des pratiques empiriques situables dans le temps et l'espace, mais également au travers de leurs significations sociales. Dans les villages jàana, elles sont intimement liées à une construction géographique des identités de sexe et de genre. Ce faisant, par l'analyse successive de décès féminins et masculins, cet article soulignera que la mobilité et l'immobilité doivent être appréhendées au pluriel : il existe des mobilités et des immobilités féminines et masculines dont les variations éclairent la localisation et la configuration contingentes des frontières spatiales de la parenté en dehors de la maison. Les mobilités et les immobilités féminines et masculines font ainsi de la maison un carrefour, soit un lieu dont les espaces intérieurs sont reliés à de multiples lieux extérieurs à l'échelle du village, de la région ou du pays. Toutefois, l'identité de ces lieux extérieurs explique les aléas et les conflits inhérents à la production de l'alliance et de la parenté dans l'espace et le temps. Elle éclaire la propension de la maison à reproduire les frontières parentélaires en dehors de son enceinte en situant la parenté dans un espace morcelé. Elle informe également d'autres recompositions possibles des frontières spatiales de la parenté en révélant le risque d'effondrement pesant sur la maison du fait de mobilités et d’immobilités féminines et masculines déstructurantes.

\section{Le chemin de l'alliance, la voie du rapt}

Parmi les dix-sept morts féminines recensées, le contingent le plus important de décès concerne six veuves âgées (35\%). Cinq de ces veuves sont mortes parce que, a-t-on dit lors de leurs funérailles, « le grenier les a tuées » : gborgu hu. En effet, lors de l'interrogatoire de leur cadavre, elles ont conduit les fossoyeurs devant le grenier que leur mari avait construit pour elles et où il entreposait les récoltes de la maisonnée. Leur mari disparu, elles ont été délaissées par leurs fils qui peinaient à subvenir aux besoins de mère, épouse(s) et enfants.

La mort de ces veuves « tuées par le grenier » est perçue comme « naturelle » du fait de l'âge avancé des défuntes. Elle rappelle que les femmes persistent dans le monde grâce à des hommes nourriciers, dont la disparition est pour elles annonciatrice de mort. En tant qu'épouses, elles obtiennent leur principale subsistance en participant à leur production dans les champs et les jardins de leur époux et cèdent leur corps dans le cadre de la sexualité afin qu'elles prolongent les lignées (Tabet, 2004). En tant que mères, elles subissent les contraintes économiques et matérielles pesant sur leur descendance dès qu'elles deviennent physiquement incapables au travail. Il n'est ainsi pas rare que les fils et les belles-filles de ces veuves considèrent que la nourriture accaparée par ces aînées est « cadeau » (pha), c'est-à-dire gâchée et dilapidée pour des femmes qui restent « assises en attendant la mort » (wua cie tan sile kiro).

Ces décès montrent que la maison est le lieu de conflits entre époux et épouse, mère et fils, belle-mère et belle-fille. Ces conflits ont une dimension économique et sexuelle 
en confrontant des hommes, propriétaires des demeures, des champs et des jardins, à des femmes arrivées sous l'effet de la résidence patri-virilocale et fournissant leur force de travail pour la maisonnée. C'est pourquoi il est dit, dans les villages jàana, que les femmes " n'ont pas de maison, mais un dedans » (khara wua a dãa wa, võro wua ama) où elles sont accueillies par des hommes. Les conflits opposent également belle-mère et belle-fille. Les premières font supporter le poids d'une dette filiale dont pâtissent les secondes qui, elles, demeurent insérées dans des échanges économiques et sexuels. Toutefois, cette lecture économique et sexuelle admet une traduction spatiale. D’un côté, les veuves sont effectivement « assises » dans la maison où elles ont été immobilisées par l'âge. Par contraste, les belles-filles se distinguent par leurs mobilités en allant aux champs et aux jardins de leur époux, en prenant en charge les activités domestiques dans la maison, en s'attelant à la production d'une lignée dans la chambre, etc. De l'autre côté, les hommes nourriciers, père comme fils, se transmettent maisons, cours, champs et greniers en ligne agnatique. Ils complètent parfois les ressources de la maison en pratiquant la pêche et la chasse dans la brousse environnante et, par le passé, en allant travailler, pendant la saison sèche, dans les plantations ivoiriennes situées à moins de $400 \mathrm{~km}$ des villages. Bien qu'en mouvement, ils n'en sont pas moins contraints à une immobilité idéale : ils doivent rester dans des lieux définissant une masculinité fondée sur la production de richesses.

La mort de ces cinq veuves est heuristique de plusieurs points de vue. D'abord, elle témoigne de l'infériorisation des femmes immobiles, notamment des veuves ménopausées que l'âge et la maladie ont figées dans la maison, comparativement aux hommes immobiles et aux femmes mobiles. Non seulement ces veuves sont incapables d'être mobiles pour effectuer le travail attaché à leur sexe, mais elles sont aussi extraites des mobilités matrimoniales et sexuelles compte tenu de leur infécondité. Dès lors, leur inertie dans l'espace signale qu'elles ne reproduisent plus la vie et ne participent plus à la production des richesses pour la maison. Ensuite, la mort de ces cinq veuves renseigne en négatif sur l'attachement de l'identité masculine à l'immobilité, laquelle atteste du pouvoir nourricier des hommes et de leur capacité à accumuler des richesses. Enfin, elle informe par contraste du rôle positif joué par les mobilités des femmes. Elles se traduisent par leur nécessaire circulation dans le cadre de l'alliance, mais aussi par les mouvements générés par les activités des épouses. Toute entrave à ces mobilités féminines peut être létale, notamment pour les hommes (6\%) décédés parce qu'ils n'ont pas obtenu de nouvelle épouse après la mort de leur première femme et sont restés célibataires jusqu'à en trépasser.

Si la défaveur va aux femmes immobiles plutôt qu'aux femmes mobiles, les secondes n'en forment pas moins un groupe apparemment dominé. Quand la mort des femmes n'est pas liée au veuvage, leur décès est le plus souvent présenté comme une sorte d'«auto-assassinat » (35\%), soit comme une mort qui, sans être désignée comme un suicide, aurait été provoquée par la défunte elle-même. Ainsi une épouse est-elle morte parce qu'elle s'est convertie à la suite de son époux à l’islam, qui est perçu comme une reli- 
gion sorcière dans les villages jàana, en dépit de la désapprobation de sa famille. Une autre est décédée, en provoquant la mort d'une autre femme de sa cour, parce qu'elle a délibérément endommagé l'autel du dieu de la guerre Dahuru de son mari qu'elle soupçonnait d'infidélité. Une troisième femme est morte parce qu'elle n'a pas avoué à son mari qu'elle avait pris du mil dans le grenier pour préparer le dîner et, ce dernier pensant que des voleurs en voulaient à ses récoltes, y a secrètement placé la corne du dieu de la pluie Hunu afin qu'il punisse les coupables. Une quatrième femme a trouvé la mort en donnant par inadvertance à son époux des viandes sacrées qui devaient revenir au chef de la société initiatique. Enfin, deux femmes sont décédées fauchées par un camion parce qu'elles ont été infidèles à leur époux, l'une d'elles entraînant également son enfant dans la mort. Ces explications de la mort, toutes produites au cours des rituels funéraires, dépeignent les femmes comme désobéissantes, soupçonneuses, négligentes, menteuses, voleuses et infidèles, dont l'inconséquence justifie qu'elles soient dominées. Leur subordination peut s'expliquer par l'existence d'une « valence différentielle des sexes » (Héritier, 2012 :17-18) prenant racine dans la biologie de la procréation et soutenant l'échange des femmes par les hommes. Elle admet toutefois une traduction spatiale, au travers de l'effort, mis en œuvre par des agents immobiles, pour capter et fixer des femmes mobiles dans l'espace de la maison afin qu'elles y reproduisent la vie et les groupes de parenté et s'attellent au travail lié à leur sexe.

Le rapt rituel, anciennement pratiqué par les Jàana et décrit par Henri Labouret (1931: 270), illustrait de manière exemplaire la force déployée par des agents immobiles pour conduire physiquement les femmes mobiles sur le chemin de l'alliance et les fixer dans une demeure. Le rapt ritualisait un combat opposant un fiancé à sa compagne, tout comme il marquait la connivence clandestine et intéressée de leurs parents respectifs. Ainsi, quinze jours après que le père de la future épouse ait reçu deux pintades - animal féminin par excellence - du père du fiancé, les amis du jeune homme se rendaient dans la demeure de la future compagne située dans la cour du père de cette dernière. L'un d'eux pénétrait dans la maison pour capturer la femme, tandis que la famille entendant ses cris sortait pour frapper les ravisseurs. Arrivée chez son fiancé, la jeune femme entrait dans sa nouvelle maison en pleurant tandis que son frère tentait de l'exfiltrer jusqu'à ce que les parents du fiancé lui aient offert de la bière de mil et un coq. Chaque ordre donné à la nouvelle épouse (se laver, s'asseoir, boire, balayer, puiser de l'eau ou chercher du bois) par sa belle-famille donnait lieu à des négociations au terme desquelles elle recevait des cauris. Pendant cinq jours, non seulement la famille de la fiancée devait refuser de quitter la nouvelle demeure de leur parente captive, mais les amis du fiancé donnaient au jeune homme des volailles pour qu'il les sacrifie et les remette à sa compagne. Lorsque la famille de la fille consentait finalement à partir à force de dons, la sœur du fiancé devait rester aux côtés de la fiancée jusqu'au retour du conjoint à la nuit tombée. Ils passaient alors leur première nuit ensemble, mais la fiancée devait crier si son conjoint l'approchait. Le lendemain matin, les amis du fiancé constataient de la lutte que les deux époux s'étaient livrée pendant la nuit. Si la violence du rapport 
sexuel attestait de la virginité des époux, les genoux écorchés du mari témoignaient de la brutalité du combat mené par un homme intrépide face à une femme hostile. Un mois plus tard, la jeune femme revenait chez son père, où elle restait entre deux et trois mois, avant de repartir chez son mari chargée de présents.

Comme la mort des cinq veuves, le rapt rituel manifeste la soumission des femmes au travail et à la sexualité en rappelant l'intrication de leur sexe à des mobilités dans l'espace. Devant ces femmes mobiles se dressent des agents immobiles attachés à des maisons alliées, soit des groupes de parenté complices et des hommes déterminés à les faire céder par la force. Ces agents immobiles placent les femmes mobiles sur le chemin de l'union afin de les fixer dans une demeure. Du point de vue des femmes, ce chemin met en réseau une maison paternelle avec celle d'un conjoint et d'un groupe d'affins. Par leurs mouvements, ces femmes relocalisent, dans une résidence extérieure, les frontières spatiales de leur parenté et perpétuent celle de leur conjoint dans sa propre maison où elles mettent en œuvre de nouvelles mobilités liées à leur statut d'épouses. Toutefois, ce chemin n'en demeure pas moins idéal. L'analyse, dans la partie suivante, du décès d'une sixième et dernière veuve va illustrer les vicissitudes et les rivalités pouvant contrecarrer un projet d'union. Ce décès rappellera les tensions opposant veuves immobiles et femmes mobiles, tout comme il montrera que la voie suivie par les secondes peut s'avérer être une bifurcation redéfinissant les frontières spatiales de la parenté maternelle et paternelle d'une épouse.

\section{Une excursion en maison « rouge "}

Parmi les décès des veuves, celui d'une femme du village de Kpedya fait figure d'exception. Nourrie par ses fils, ses belles-filles et les enfants de son frère résidant dans le village voisin de Sedongio, elle était contrainte, depuis de nombreuses années, à demeurer immobile dans sa cour du fait de son âge et de graves problèmes de santé. Toutefois, à sa mort, ce n'est pas son statut de veuve « tuée par le grenier » qui a expliqué son trépas. Les rites funéraires ont conclu qu'elle est morte « contrariée » par l'union de sa petite-fille, $\mathrm{Obi}^{4}$.

Sous l'autorité de sa grand-mère maternelle depuis la mort précoce de sa mère, Jbi a habité plusieurs années dans la ville de Bobo-Dioulasso avant de revenir à Kpedya. Or, quelques semaines après son retour, elle s'est installée dans la maison d'un jeune homme, Olle, qu'elle avait rencontré quelques mois plus tôt en ville. Olle est un des fils de l'adjoint du chef de terre du village de Sedongio, où réside le père de la jeune femme. Comme de nombreux jeunes villageois, il s'adonnait à l'orpaillage dans les mines d'or artisanales, qui ont essaimé aux alentours des villages jàana, afin d'accumuler un capital suffisant pour construire sa maison et participer à l'achat d'une moto avec ses frères aînés.

\footnotetext{
${ }^{4}$ Les prénoms ont été modifiés et les toponymes ont été traduits en langue jàan afin de préserver l'anonymat de ceux qui ont été impliqués dans ce conflit familial.
} 
Le déménagement soudain de Jbi chez son fiancé a fait courir le bruit qu'elle était sans doute enceinte. Il a surtout conduit la grand-mère maternelle à s'insurger contre sa petite-fille. L'aînée a envoyé à plusieurs reprises à Sedongio son propre fils, oncle utérin de Jbi, pour rompre l'union sans délai. En effet, si l'attractivité de Olle pouvait tenir à l'argent qu'il avait pu épargner ${ }^{5}$, l'union contrecarrait le projet matrimonial imaginé par la grand-mère. La grand-mère voulait unir $\mathrm{Obi}$ au fils de l'oncle maternel de cette dernière, soit à un cousin matrilatéral. Cette alliance présentait un double avantage aux yeux de l'aînée : elle permettait au jeune homme de sortir du célibat et elle s'opérait à l'intérieur du matrilignage en le stabilisant dans une maison à Kpedya. Par ailleurs, l'alliance de Jbi et Olle offensait d'autant plus la grand-mère que l'époux était un conjoint controversé. Olle appartient au groupe des « gens rouges » (ĩmbi-sya), qui est porteur de la souillure mythique de l'inceste. Autrefois, les « gens rouges » étaient contenus au sein d'un clan spécifique, les Kmaani Sua, et d'un village particulier, Mule Dãa, dont le nom rappelle le stigmate mythique ${ }^{6}$. Les hommes et les femmes « rouges » étaient contraints de s'unir dans l'espace de leur propre village, ce qui assignait de ce fait le clan à l'endogamie, afin que la " souillure " rouge n'entache pas les lignées d'autres villages. La transgression progressive de ces interdits explique qu'avec le temps des lignées « rouges » soient apparues dans d'autres groupes de parenté et d'autres maisons, notamment à Sedongio.

La mort par « contrariété » de la grand-mère rappelle le rôle de la mobilité des femmes dans la production de la vie, des relations et des groupes de parenté dans les maisons. Elle révèle toutefois la concurrence qui entoure leur captation en mettant aux prises différents agents et groupes immobiles. En effet, dans les villages jàana, les femmes transmettent les appartenances en inscrivant leur descendance dans un matrilignage et l'une des moitiés du matriclan : les « nobles » puore qui gouvernent les chefferies de clan, de village et de société initiatique et les descendants d'esclaves yawa (sing. yawo). En se refusant à son cousin matrilatéral, Jbi a donc entravé la stabilisation de son groupe maternel dans le village de Kpedya. Si tel avait été le cas, sa descendance aurait été, du côté maternel comme paternel, membre du même matrilignage. En préférant Olle, Jbi a fait émerger une lignée à tout jamais "souillée » au sein de son groupe maternel tout en participant à l'expansion sociale et spatiale d'un groupe jugé vicié. Par ailleurs, son union a réactivé un conflit entre, d'un côté, une grand-mère et des maternels ligués à elle et, de l'autre, un père et son lignage. Une tension ancienne opposait le groupe des maternels dirigés par la grand-mère et le père de la jeune femme. Ce dernier avait été accusé d’avoir provoqué la mort de sa première épouse, qui a mis Jbi

\footnotetext{
${ }^{5}$ Cette attractivité témoigne d'une métamorphose de la virilité dans les villages jàana (Cros, Mégret, 2009).

${ }^{6}$ Bien que cette traduction soit contestée par les habitants de Mule Dãa, le toponyme viendrait du verbe « e miiye dãa » qui signifie « la maison qui prend feu ». Cette traduction se réfère explicitement à l'inceste car, pour décrire l'identité jugée corrompue des « hommes rouges », il est dit que leur «pénis enflamme les femmes », qu'ils les désirent toutes, même celles qui leur sont interdites : wua gbiere ko mulo dĩe dãa («leur pénis enflamme la maison ») ou ko gbiere le mulo dĩe (" leur pénis brûle comme le feu »).
} 
au monde. Or sa défunte femme était sa cousine matrilatérale, soit la fille (bi-kheru) de la grand-mère " contrariée » et la nièce utérine $(v m u)$ du père $(j a)$ dans la cour duquel l'homme réside.

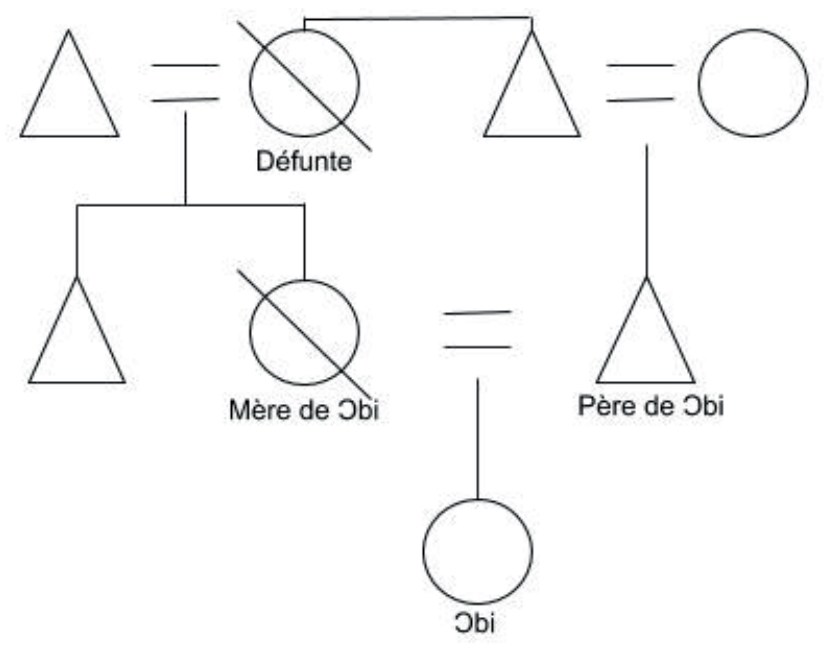

Figure 1. Liens de PARenté entre LA GRAND-MÈre défunte et LE PÈre de つBI

De plus, le père, loin de s'émouvoir de l'union de Obi et Olle, y a vu une opportunité. Il entretient, du côté maternel, des relations de parenté avec le groupe dans lequel elle s'est mariée : la sœur cadette de sa mère (sa "petite-mère », na-bu) a épousé le frère aîné (kinu) du père de Olle. L'alliance de sa fille Jbi lui a donc permis de consolider ses liens avec des maternels de Sedongio dont il a assuré la pérennisation sociale et spatiale en enjoignant sa fille à demeurer dans leur maison.

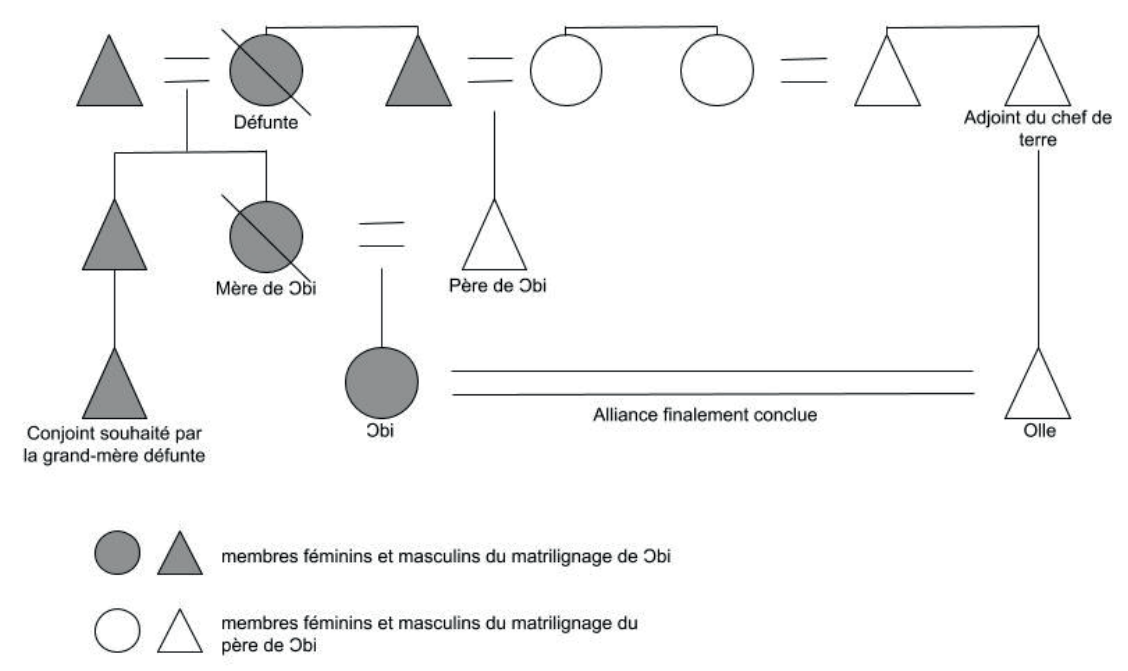

Figure 2. L'alliance de गbi et de Olle 
Si la grand-mère a succombé devant cette alliance et le père s'en est réjoui, tous deux pouvaient légitimement imposer leur autorité sur Jbi. En effet, dans les villages jàana, les règles d'alliance autorisent l'union de la première fille de la descendance avec le fils (bi-uo) de l'oncle maternel (jicnu) de cette dernière et celle de la seconde fille avec le neveu utérin $(\mathrm{vmu})$ de son propre père $(\mathrm{ja})$. Il était donc légitime que le père appuie l'alliance de sa fille Jbi à Sedongio, tout autant qu'il était légitime que la grand-mère maternelle, ayant vu sa première petite-fille Yeli épouser un homme étranger à son matrilignage, désire unir Jbi à un cousin matrilatéral de Kpedya.

En demeurant finalement avec Olle, Jbi a redéfini les frontières spatiales de sa parenté maternelle et paternelle. Par son refus d'une alliance endogène, Jbi a empêché la perpétuation de son groupe maternel à Kpedya et délocalisé son matrilignage dans une demeure "impure » et extérieure à sa cour et à son village maternels. Cette union controversée montre donc que la mobilité des femmes peut constituer le lieu de la connivence d'agents immobiles plaçant les femmes sur un chemin matrimonial prédéterminé et idéal, mais aussi celui de rivalités entre d'autres agents immobiles qui cherchent à s'en emparer pour les fixer dans la demeure d'un époux et la cour d'un groupe receveur. L'union de Jbi révèle ainsi que les femmes peuvent suivre des voies matrimoniales permettant la production des frontières spatiales de la parenté en actualisant des liens entre des maisons. Elles peuvent également exciter des conflits entre des maisons maternelles et paternelles en menaçant l'ancrage des premières au profit des secondes. Sur les voies que les femmes empruntent se jouent donc des rapports de force entre des agents immobiles, où l'argent apparaît, qui plus est, comme un pôle d'attraction et de détournement des femmes mobiles.

Cette excursion en maison " rouge " incarne une forme différentielle de mobilité féminine qui existe aux côtés d'un autre type de mobilité tel qu'il était ritualisé dans le rapt. Elle s'explique toutefois au regard d'autres phénomènes de circulation qui peuvent être également conçus en termes spatiaux : l'envoi de filles en ville, le départ des jeunes hommes dans les mines et la circulation de l'argent de l'or. L'abandon du rapt rituel et de l'excision ont, de leur côté, accru l'image de mobilités féminines incontrôlables dans les villages jàana. Ces transformations expliquent qu'il est dit des jeunes filles non excisées qu' « elles cherchent les hommes" (wua kaale ua) et qu'une chanson de réjouissance ${ }^{7}$ annonce que « de nos jours, une jeune fille, si tu lui tends de l'argent, elle couche avec toi ! (moara dagbi-khar, wua ni-go, 'le fa-o !). Que les mobilités des femmes profitent à certains agents immobiles plutôt qu'à d'autres, elles participent, dans tous les cas, à la recomposition des frontières spatiales de leur parenté maternelle et paternelle. La parenté se distribue ainsi, dans la contingence, dans un espace géographique morcelé en étant le résultat de mobilités féminines qui perpétuent ou bien inquiètent la reproduction de maisons d'agents immobiles.

\footnotetext{
${ }^{7}$ Les chansons de réjouissance sont interprétées par les femmes, qui sont accompagnées d'hommes jouant du balafon, de longs tambours et de tambours d'aisselle, lors de festivités comme le Nouvel An ou d'inaugurations de bâtiments publics contre rémunération.
} 
L'étiologie de la mort de ces six veuves montre que des mobilités féminines et des immobilités masculines configurent de manière dynamique les frontières spatiales de la parenté. Elles mettent en réseau maisons, cours, villages, villes et mines et produisent, dans l'aléa du temps, l'espace de la parenté en localisant ses frontières dans certaines demeures. À côté de ces mobilités féminines, se font également jour des immobilités féminines dans la maison, telles celles des veuves ménopausées. Ces immobilités sont, elles, socialement dévalorisées dans la mesure où elles contredisent la définition géographique des identités de genre. La partie suivante présentera d'autres variations possibles de mobilités et d'immobilités féminines et masculines en montrant qu'il existe des mobilités féminines à l'origine de l'immobilisation létale d'époux. Elles éclairent autrement les reconfigurations possibles des frontières spatiales de la parenté en présageant, cette fois, de la menace d'effondrement pesant sur la maison et de la (re)mise en mouvement possible de ses habitants.

\section{Les lieux masculins de l'exil}

Les trente-et-un décès masculins recensés distinguent deux causes principales de la mort des hommes : ce sont, d'abord, l'argent et les circulations monétaires (39\%) et, enfin, la sexualité avec les femmes (32\%). La pratique de la sorcellerie (14 \%), l'« auto-assassinat » $(9 \%)^{8}$ et le célibat (6\%) sont, comparativement, des explications secondaires de décès. Dans le cas où la sexualité est à l'origine de la mort des hommes, les femmes sont toujours rendues coupables des décès'. Loin d'être seulement un groupe dominé par des agents immobiles, les femmes forment en cela une " moitié dangereuse » (Balandier, 1985) dotée du pouvoir de tuer les hommes du fait de leurs mobilités. L'analyse des décès masculins qui suit va montrer que ce pouvoir féminin tient à la capacité des femmes à mettre les hommes en mouvement pour finalement les immobiliser dans des lieux dangereux extérieurs à la maison.

Les femmes accusées des morts masculines seraient à l'origine d'une transgression ou d'une frustration sexuelle mortelle pour les hommes. Ainsi, un premier ensemble de décès masculin ( $40 \%$ ) concerne les devins ( $k$ sla ja, sing. $k\lrcorner l s j a)$. L’initiation à la divination oblige les devins et leurs épouses à respecter des interdits sexuels dont la violation est sanctionnée par la mort. Un devin ne peut avoir de rapport sexuel avec une femme en dehors de sa natte et de sa chambre. Son épouse, quant à elle, ne peut lui être infidèle ou refuser trois fois de suite d'avoir un rapport sexuel. Kolo règle ainsi de manière drastique la sexualité des initiés et de leurs compagnes. Or les transgressions sexuelles tuant les devins, notamment les rapports sexuels en dehors de la chambre de l'initié et les adultères, sont désignées par deux expressions synonymes : on dira du ou

\footnotetext{
${ }^{8}$ Les trois seuls cas d'« auto-assassinat » masculin sont liés à des conversions controversées au protestantisme évangélique et à l'islam, et aux velléités d'un frère qui aspirait à destituer son aîné de la chefferie de terre du village.

${ }^{9}$ De manière générale, les hommes sont le plus souvent présentés comme des victimes de meurtre à leur mort, soit parce qu'ils s'entretuent dans des cycles de vendetta liés aux aléas des circulations monétaires, soit parce qu'ils sont exécutés par une femme.
} 
de la coupable qu' « il/elle est parti(e) en brousse » (li-u brngo) ou encore que « la natte est devenue étrangère » (kpala 6aaye). C'est pourquoi il ou elle a l'interdiction de pénétrer physiquement dans l'enceinte domestique et de manger le mil du grenier sans avoir avoué son forfait. Sa présence menacerait l'intégrité de la maison, dans la mesure où le ou la coupable est accusé(e) d'avoir frayé une bifurcation mortelle reliant directement l'enceinte domestique, notamment la chambre conjugale et les greniers, ainsi que les champs des hommes à la "brousse », des espaces contraires bien qu'inséparables (Cartry, 1979). Les expressions " partir en brousse " et " natte étrangère » attribuent ainsi la mort des hommes à une mobilité féminine létale, soit parce qu'un devin a été immobilisé hors de chez lui par une femme, soit parce qu'une épouse s'est détournée du chemin la menant chez son époux pour retrouver son amant.

Un second ensemble de morts masculines (30 \%) attribue le décès des hommes à des dépenses d'argent pour des femmes séduisantes rencontrées dans les marchés, les buvettes et les maquis ${ }^{10}$ qui abondent désormais aux abords des pistes et des routes. Les hommes s'y sont attardés pour acheter boisson et nourriture en espérant obtenir les faveurs de ces femmes, tandis que ces dernières y ont entraîné les hommes pour obtenir d'eux des cadeaux. Mais, à force de dépenses, ces derniers se sont retrouvés sans le sou et sont devenus incapables de satisfaire les dieux et les ancêtres qui les appelaient pour qu'ils réalisent des sacrifices dans leur maison, leur cour ou leurs champs. Comme pour les devins, ces morts admettent une traduction spatiale. Les femmes sont rendues coupables d'avoir détourné les hommes des dieux et des ancêtres les rappelant à la maison ou aux champs pour les faire demeurer physiquement « au dehors " (kiaalo), dans les marchés, les buvettes et les maquis. Les hommes sont donc morts parce qu'ils sont objectivement restés au bord de voies et de pistes, dans une sorte d'entre-deux spatial à mi-chemin entre la maison, la cour et les champs, sur lesquelles des femmes mobiles les ont attirés. En ce sens, l'identité de ces femmes des marchés, des buvettes et des maquis est intimement liée à la route (Masquelier, 2002) ${ }^{11}$ parce qu'elles déracinent les hommes de lieux où ils doivent idéalement revenir pour y demeurer immobiles.

Qu'il s'agisse des devins ou des hommes ruinés, les morts masculines trouvent leur origine dans un exil provoqué par une maîtresse, une épouse infidèle ou une femme séduisante. Ces femmes ont soit ouvert des voies dangereuses, soit immobilisé les hommes sur des routes bifurquant à l'égard de celles conduisant vers leur demeure et leurs champs. Cet exil masculin se matérialise donc par une immobilisation létale qui est, de surcroît, à l'origine de reconfigurations nouvelles des frontières spatiales de la parenté. En effet, ces époux décédés ont fait peser une incertitude quant à l'avenir de leur maison. Tout en exposant leur demeure à des incursions et des attaques surnaturelles venant de la brousse ou de dieux et d'aïeuls courroucés, leur trépas présage du

${ }^{10}$ Ce sont des lieux de restauration dont le nom, issu du français, désigne un espace de fuite à la végétation dense et difficilement accessible.

${ }^{11}$ Ces femmes sont comparables aux maîtresses des salariés et des fonctionnaires, nommées « go » ou « deuxième bureau » (Lacombe, 1983), et à la figure de la prostituée dont le nom, « naakhar » (« femme-vache »), l'érige comme l'archétype de la femme nomade qui, comme le bétail, erre dans la brousse pour se nourrir. 
délitement de leur maison. Leur mort pose de multiples questions : la veuve pourrat-elle se remarier dans une autre demeure ou devra-t-elle retourner dans sa maison d'origine ? À qui les enfants seront-ils confiés ? Les décès de ces époux provoquent des mobilités et des immobilités accidentelles en générant une (re)mise en circulation fortuite des femmes et des enfants de la maison et, de ce fait, la production de nouvelles frontières spatiales de la parenté.

\section{Conclusion}

Pour conclure, les formes plurielles de mobilités et d'immobilités féminines et masculines délimitent, dans la contingence du temps, les frontières spatiales de la parenté en dehors de la maison. En ramifiant l'intérieur de la maison à des lieux extérieurs, elles font de l'espace domestique un carrefour, soit une zone " ouverte » de contacts et de mouvements. À ce carrefour se jouent les multiples reconfigurations possibles des frontières spatiales de la parenté, dont l'issue tient à l'identité des lieux extérieurs auxquels la maison se trouve reliée : est-ce une maison alliée, controversée ou rivale, la demeure d'une maîtresse ou d'un amant, un marché, une buvette ou un maquis ? La mise en réseau de la maison avec ces lieux peut permettre l'établissement de liens durables entre des groupes en stabilisant la parenté d'immobiles dans des maisons et en la localisant dans un espace morcelé. Elle peut aussi augurer de l'effondrement d'une demeure par la fixation conflictuelle d'une fille dans une maison plutôt qu'une autre ou par des immobilités masculines funestes causant la fuite accidentelle de femmes, d'enfants et de parents. Ainsi, la localisation contingente des frontières spatiales de la parenté témoigne de l'inscription de la maison dans un territoire historique en transformation, marqué par des circulations de personnes, de biens et de richesses dans l'espace et le temps. Il resterait toutefois à déterminer dans quelle mesure la maison, considérée du point de vue de son architecture et de sa topographie, modélise à son tour l'existence d'une frontière spatiale symbolique figurant la rencontre toujours conflictuelle entre des femmes mobiles et des hommes immobiles.

\section{Bibliographie}

Balandier G. (1985 [1974]), « Hommes et femmes ou la moitié dangereuse », in Anthropologiques, Paris, Librairie Générale Française, p. 31-84.

Bergeon C., Dureau F., Imbert C., Le Roux G., Lessault D. (2013), « Et l’immobilité dans la circulation? », e-migrinter, $\mathrm{n}^{\circ} 11, \mathrm{p}$. 3-6.

Carsten J., Hugh-Jones S. (dir.) (1995), About the house. Lévi-Strauss and beyond, Cambridge, Cambridge University Press.

CARTRY M. (1979), « Du village à la brousse ou le retour de la question. À propos des Gourmantché du Gobnangou (Haute-Volta) » in Izard M., Sмith P. (dir.), La fonction symbolique : essais d'anthropologie, Paris, Gallimard, p. 265-288. 
Cros M., Megret Q. (2009), « D’un idéal de virilité à l'autre ? Du vengeur de sang au chercheur d'or en pays lobi burkinabé », Autrepart, vol. 49, n 1, p. 137-154.

Dessertine A. (2016), Présences imminentes. Mobilités et production des espaces dans un village malinké de Guinée, thèse de doctorat, Paris, Université Paris-Nanterre.

HAMberger K. (2010), " La maison en perspective. Un modèle spatial de l'alliance », L'Homme, vol. 194, n² 2, p. 7-40.

HÉRITIER F. (2012 [2002]), Masculin/Féminin II. Dissoudre la hiérarchie, Paris, Odile Jacob.

Labouret H. (1931), Les tribus du rameau lobi, Paris, Institut d'Ethnologie.

LACомBE B. (1983), « Le deuxième bureau. Secteur informel de la nuptialité en milieu urbain congolais », STATECO, n 35, p. 37-57.

LÉVi-Strauss C. (1984), Paroles données, Paris, Plon.

Masquelier A. (2002), " Road mythographies: space, mobility, and the historical imagination in postcolonial Niger », American Ethnologist, vol. 29, n 4, p. 829-856.

ReEves M. (2011), « Staying put? Towards a relational politics of mobility at a time of migration », Central Asian Survey, vol. 30, n³-4, p. 555-576.

SCHEWEL K. (2019), « Understanding immobility: Moving beyond the mobility bias in migration studies », International Migration Review, vol. 20, n 10, p. 1-28.

TABET P. (2004), La grande arnaque. Sexualité des femmes et échange économico-sexuel, Paris, L’Harmattan. 